\title{
子符号差算子及其局部指标定理 ${ }^{*}$
}

张伟平

(南开数学研究所, 天津 300071)

\section{关键词 子符号差算子 局部指标 示性式及示性类}

设 $M$ 是一紧致无边的定向微分流形, 设 $E$ 为 $M$ 的一个定向切子丛, 我们假定 $k=\operatorname{dim} E$ 为偶数.

设 $g^{T M}$ 为切丛 $T M$ 上的一个度量, 记 $E^{\prime}$ 为 $T M$ 中关于 $g^{T M}$ 的正交补. 记 $g^{E}$ 及 $g^{E}$ 为 $g^{T M}$ 在 $E$ 及 $E^{\prime}$ 上的限制, 则 $T M$ 有正交分解 $T M=E \oplus E^{\prime}, g^{T M}=g^{E} \oplus g^{E^{\prime}}$, 并且 $E^{\prime}$ 上有自然的诱导定 向.

令 $\Lambda\left(T^{*} M\right)=\bigoplus_{i=1}^{\operatorname{dim} M} \Lambda^{i}\left(T^{*} M\right)$ 为 $M$ 的复系数外代数丛. 记 $\Omega(M)=\bigoplus_{i=0}^{\operatorname{dim} M} \Omega^{i}(M)=\bigoplus_{i=0}^{\operatorname{dim}}{ }^{M} \Gamma$ $\left(\Lambda^{i}\left(T^{*} M\right)\right)$ 为 $\Lambda\left(T^{*} M\right)$ 的光滑截影全体, 则 $g^{T M}$ 在 $\Lambda\left(T^{*} M\right)$ 及 $\Omega\left(T^{*} M\right)$ 上有自然的诱导度 量和内积.

熟知 $T M$ 及 $T^{*} M$ 在 $g^{T M}$ 下等价. 对任何的 $e \in \Gamma(T M)$, 令 $\hat{c}(e)=e \Lambda+i_{e}$, 其中 $e \Lambda, i_{e}$ 分 别是 $e \in T^{*} M \simeq T M$ 在 $\Omega(M)$ 上的外乘积及内乘积作用. 设 $f_{1}, \cdots, f_{k}$ 为 $E$ 的一组 (局部) 定向 么正基. 令

$$
\hat{c} \partial\left(E, g^{E}\right)=\hat{c}\left(f_{1}\right) \cdots \hat{c}\left(f_{k}\right)
$$

易证 $\hat{c}\left(E, g^{E}\right)$ 不依赖于么正基 $\left\{f_{i}\right\}_{1 \leqslant i \leqslant k}$ 的选取.

记 $\varepsilon \in$ End $\left(\Lambda\left(T^{*} M\right)\right)$ 使得 $\left.\varepsilon\right|_{\Lambda \text { even }\left(T^{*} M\right)}=I d,\left.\varepsilon\right|_{\Lambda \text { odd }\left(T^{*} M\right)}=-I d$.

命

$$
\tau\left(M, g^{E}\right)=(\sqrt{-1})^{-\frac{k(k+1)}{2}} \varepsilon \hat{c}\left(E, g^{E}\right)
$$

则经验算有 $\tau\left(M, g^{E}\right)^{2}=I d$.

令 $\Lambda \pm\left(T^{*} M, g^{E}\right)$ 为由下式决定的 $\Lambda\left(T^{*} M\right)$ 的子丛:

$$
\Gamma\left(\Lambda \pm\left(T^{*} M, g^{E}\right)\right)=\left\{\omega \mid \omega \in \Gamma\left(\Lambda\left(T^{*} M\right)\right), \tau\left(M, g^{E}\right) \omega= \pm \omega\right\} \text {. }
$$

记 $\Omega^{ \pm}\left(M, g^{E}\right)=\Gamma\left(\Lambda \pm\left(T^{*} M, g^{E}\right)\right)$ 为 $\left.\Lambda^{ \pm}\left(T^{*} M, g^{E}\right)\right)$ 的光滑截影全体.

记 $d$ 为 $\Omega(M)$ 上的外微分算子, $\delta$ 为 $d$ 关于 $\Omega(M)$ 上自然诱导内积的 (形式) 伴随算子. 令 $D_{E}$ 为下式给出的 $\Omega(M)$ 上的微分算子:

$$
D_{E}=(\sqrt{-1})^{\frac{k(k+1)}{2}} \frac{1}{2}\left(\hat{c}\left(E, g^{E}\right)(d+\delta)+(d+\delta) \hat{c}\left(E, g^{E}\right)\right) .
$$

容易验证, $D_{E}$ 是 $\Omega(M)$ 上的一阶 (形式) 自伴椭圆微分算子, 并且将 $\Omega_{ \pm}\left(M, g^{E}\right)$ 互换.

1995-04-12 收稿, 1995-08-12 收修改稿

*国家教育委员会及国家自然科学基金资助项目 
定义 $D_{E}$ 在 $\Omega_{+}\left(M, g^{E}\right)$ 上的限制称为 $\left(E, g^{T M}\right)$ 的子符号差 (sub-signature) 算子, 并记为 $D_{E,+}$. 它把 $\Omega_{+}\left(M, g^{E}\right)$ 映到 $\Omega_{-}\left(M, g^{E}\right)$.

记 $\exp \left(-t D_{E}^{2}\right)$ 为 $D_{E}^{2}$ 的热算子, 记 $d V_{M}$ 为 $g^{T M}$ 决定的 $M$ 上的体积元. 命 $p_{t}(x, y)$ 为 $\exp$ $\left(-t D_{E}^{2}\right)$ 关于 $d V_{M}$ 的核.

回顾一下, $\tau\left(M, g^{E}\right)$ 决定了 $\Lambda(M)$ 上的一个 $\mathbb{Z}_{2}$ - 分次结构 (也称超结构) $\Lambda(M)=\Lambda+(M$, $\left.g^{E}\right) \oplus \Lambda-\left(M, g^{E}\right)$. 记 $\operatorname{tr}_{s}^{\tau}$ 为由此分次结构决定的超迹 (supertrace).

现在命 $\nabla^{T M}$ 为 $g^{T M}$ 的 Levi-Civita 联络, 命 $\nabla^{E}$ 及 $\nabla^{E^{\prime}}$ 为 $\nabla^{T M}$ 在 $E$ 及 $E^{\prime}$ 上的限制, 并分别记 $\nabla^{T M}, \nabla^{E}$ 及 $\nabla^{E}$ 的曲率为 $R^{T M}, R^{E}$ 及 $R^{E}$.

本简报的主要结果为如下之局部指标定理.

定理 下面的收敛公式在 $M$ 上一致成立:

$$
\begin{gathered}
\lim _{t \rightarrow 0} \operatorname{tr}_{s}^{t}\left[p_{t}(x, x)\right] d V_{M}(x)= \\
\left(\frac{2}{\sqrt{-1}}\right)^{\frac{k}{2}}\left\{\operatorname{det} \frac{1}{2}\left(\frac{R^{T M} / 4 \pi}{\sinh \left(R^{T M} / 4 \pi\right)}\right) \cdot \operatorname{det} \frac{1}{2}\left(\cosh \left(\frac{R^{E}}{4 \pi}\right)\right) \cdot\right. \\
\left.\operatorname{det} \frac{1}{2}\left(\frac{\sinh \left(R^{E^{\prime}} / 4 \pi\right)}{R^{E^{\prime}} / 4 \pi}\right) \cdot \operatorname{Pf}\left(\frac{R^{E^{\prime}}}{2 \pi}\right)\right\} \max (x), \forall x \in M .
\end{gathered}
$$

由此定理, Mckean-Singer 公式以及示性类的陈省身 -Weil 理论 ${ }^{[1]}$ 立即得到下面的推论.

推论 下面的式子成立:

$$
\text { ind } D_{E,+}=\langle\mathscr{L}(E) e(T M / E),[M]\rangle \text {, }
$$

其中 $\mathscr{L}(E)$ 为 $E$ 的 Hirzebruch $\mathscr{L}$ - 示性类, $e(T M / E)$ 为商丛 $T M / E$ 的 Euler 示性类.

注 1 令 $E=0$ 或 $E=T M$, 则 (6) 式分别表现为著名的 Gauss-Bonnet- 陈省身公式和 Hirzebruch 符号差公式 ${ }^{[1]}$. 因此我们的结果统一了这两个经典定理.

- 注 2 我们可以在 $D_{E}$ 的定义中扭合 (twist)一个 $M$ 上的向量丛作为系数. 这时有相应的 扭合 (twisted) 指标定理, 它可以用来给出 $e(T M / E)$ 的解析解释.

注 3 对于 $\operatorname{dim} E$ 为奇数的情形, 有相应的奇指标定理及其局部形式.

注 4 本文内容可以应用的一类重要例子是当 $M$ 为一个纤维丛甚或叶状结构 (foliation) 的情形, 与此相关的问题将另外作讨论.

本简报的内容将在其他地方给出详细的叙述及证明.

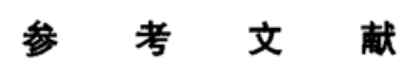

1 Berline N, Getzler E, Vergne M. Heat Kernels and Dirac Operators. Berlin: Springer-Verlag, 1992 